\title{
Detection and validation of volatile metabolic patterns over different strains of two human pathogenic bacteria during their growth in a complex medium using multi-capillary column-ion mobility spectrometry (MCC-IMS)
}

\author{
Nils Kunze • Julia Göpel • Martin Kuhns • \\ Melanie Jünger • Michael Quintel • Thorsten Perl
}

Received: 1 December 2012 /Revised: 4 February 2013 /Accepted: 7 February 2013 /Published online: 7 March 2013

(C) The Author(s) 2013. This article is published with open access at Springerlink.com

\begin{abstract}
Headspace analyses over microbial cultures using multi-capillary column-ion mobility spectrometry (MCCIMS) could lead to a faster, safe and cost-effective method for the identification of pathogens. Recent studies have shown that MCC-IMS allows identification of bacteria and fungi, but no information is available from when on during their growth a differentiation between bacteria is possible. Therefore, we analysed the headspace over human pathogenic reference strains of Escherichia coli and Pseudomonas aeruginosa at four time points during their growth in a complex fluid medium. In order to validate our findings and to answer the question if the results of one bacterial strain can be transferred to other strains of the same species, we also analysed the headspace over cultures from isolates of random clinical origin. We detected 19 different volatile organic compounds (VOCs) that appeared or changed their signal intensity during bacterial growth. These included six VOCs exclusively changing over $E$. coli cultures and seven exclusively changing over $P$. aeruginosa cultures. Most changes occurred in the late logarithmic or static growth phases. We did not find differences in timing or trends in signal intensity between VOC patterns of different strains of one species. Our results show that differentiation of human pathogenic bacteria by headspace analyses
\end{abstract}

N. Kunze $(\bowtie) \cdot J$. Göpel $•$ M. Jünger $\cdot$ M. Quintel $\cdot$ T. Perl Department of Anaesthesiology, Emergency and Intensive Care Medicine, University of Göttingen, Robert-Koch-Straße 40, 37075 Göttingen, Germany

e-mail: nkunze@gwdg.de

M. Kuhns

Institute for Medical Microbiology, University of Göttingen, Kreuzbergring 57, 37075 Göttingen, Germany using MCC-IMS technology is best possible during the late phases of bacterial growth. Our findings also show that VOC patterns of a bacterial strain can be transferred to other strains of the same species.

Keywords Escherichia coli $\cdot$ Pseudomonas aeruginosa . Bacteria $\cdot$ Identification $\cdot$ Headspace analyses

\section{Introduction}

Clinical outcome in patients with bacterial infections is strongly related to the early administration of an adequate antibiotic therapy (Kollef et al. 1999; Houck et al. 2004; Garnacho-Montero et al. 2006; Kumar et al. 2009). Confirmation of the adequacy of the antibiotic therapy initially chosen still depends on the results of conventional microbiological diagnostic methods, such as enzyme-based testing or culturing of clinical isolates. By using these methods, this important information is available after 48 to $96 \mathrm{~h}$. However, clinical studies have shown a dramatic increase in hospital mortality associated with the delay of adequate antibiotic therapy for more than $24 \mathrm{~h}$ (GarnachoMontero et al. 2006). Additional data suggest a negative influence on clinical outcome even for less delay in the initiation of an antibiotic therapy (Houck et al. 2004; Kumar et al. 2009).

Since for that reason time is running from the moment of the clinical diagnosis of an infection, many attempts have been made to provide faster microbiological testing. Recently developed methods are aiming at molecular biological or proteomic characteristics of pathogens (Yoo and Lee 2008; Mach et al. 2009; Fenollar et al. 2006; van Veen 
et al. 2010). Although these methods (e.g. DNA microarrays, multiplex PCR, MALDI-, ESI- or SELDI-TOF) offer major advantages, such as the possibility to identify the pathogens very quickly (6 h) (Yoo and Lee 2008) as well as some information about drug resistances (Zhu et al. 2007), they have not become routine diagnostic methods in the clinical setting. Besides methodical problems, this might be due to the relatively high costs and the complexity of these diagnostic tools (Yoo and Lee 2008; Mach et al. 2009; Fenollar et al. 2006; van Veen et al. 2010).

A new approach for the rapid identification of pathogens is the detection of pathogen-specific volatile organic compounds (VOCs) by multi-capillary column-ion mobility spectrometry (MCC-IMS). The fact that microbial growth is associated with the production of different VOCs is well known to biological and medical sciences (Zoller and Clark 1921; Stotzky and Schenck 1976; Kai et al. 2009). Many microbial VOCs have been described, and some of them have been shown to be specific for the respective organisms (Schulz and Dickschat 2007; Kai et al. 2009). In 2007, Schulz and Dickschat published a review on that topic and listed 346 different compounds released from various bacteria (Schulz and Dickschat 2007). Ion mobility spectrometry (IMS) provides a comparatively cheap and fast method for the detection of these bacterial and fungal VOCs with detection limits down to a range of picograms per liter (parts per trillion).

First experiences have been made in the detection of microbial VOCs by IMS (Snyder et al. 1991; Smith et al. 1997; Chaim et al. 2003) and the related technology of differential mobility spectrometry (Shnayderman et al. 2005). Coupling IMS with a multicapillary column for pre-separation facilitates the analysis of complex and humid gas samples (Ruzsanyi et al. 2005), such as human breath. With MCC-IMS, a tool is available that provides fast and reliable information on microbial metabolic compounds and enables the detection of specific VOC patterns.

Yet little is known on how the emission of VOCs from human pathogenic bacteria changes during their growth in a medium or tissue. Recent studies gave evidence that MCC-IMS is a suitable technology for detecting VOCs from several microbes growing on a complex medium that, by itself, contains a number of detectable substances as a volatile background (Jünger et al. 2012; Perl et al. 2011). It was the aim of the present study to show from which point, during bacterial growth, a specific VOC can be detected by MCC-IMS and how its signal intensity in the IMS plot changes through the growth phases. Therefore, human pathogenic strains of Escherichia coli and Pseudomonas aeruginosa were grown in a complex medium that allows rapid cultivation of a maximum variety of human pathogenic microbes.
Most studies on the use of IMS for the detection of microbes investigated single bacterial strains of several species, and authors suggest that their findings could be transferred to other strains of the same species (Maddula et al. 2009; Jünger et al. 2012; Perl et al. 2011). With our study, we aimed to assess this hypothesis. Therefore, we compared the VOC patterns of each reference strain with the results of the MCC-IMS analyses over 12 randomly collected clinical isolates of the same species from various origins (e.g. blood and urine cultures).

\section{Methods}

Growth medium and sampling of the volatile background

All bacterial strains were grown in Lysogeny Broth fluid medium (LB, Carl Roth GmbH+Co. KG, Karlsruhe, Germany). A pH of 7.2 was established at a temperature of $37^{\circ} \mathrm{C}$ with standard Tris- $\mathrm{HCl}$ buffer. Aliquots of $200 \mathrm{ml}$ of the medium were transferred into 2,000-ml culture flasks (Schott Duran, Mainz, Germany) and autoclaved for $25 \mathrm{~min}$ at a temperature of $121^{\circ} \mathrm{C}$.

In order to detect the volatile background, 12 measurements of the cleaned and autoclaved culture flasks and 12 measurements of sterile LB medium were performed. To simulate the environmental influences on the medium during the growth of the bacteria, LB samples were agitated constantly at a temperature of $37^{\circ} \mathrm{C}$ for at least $1 \mathrm{~h}$ before measurements.

In the headspace analyses of the bacterial cultures the volatile background, consisting of compounds related to the culture flask and the fluid medium, was compared to the volatile profiles detected at four typical time points during bacterial growth. Compounds that belonged to the volatile background and which showed no change in signal intensity during the growth phases were neglected. Newly occurring signals were considered to be useful for the differentiation of the particular bacterial strain as well as signals that showed an increasing or a decreasing trend in signal intensity. If its intensity was increasing, a signal was suggested to be a bacterial metabolite. Substrate consumption from the growth medium may result in decreasing signal intensities. Therefore, we considered these signals as well to be helpful for bacterial differentiation and analysed them for the present study.

Bacterial strains, culturing and growth

One strain of $E$. coli (DSM 25944) and one strain of $P$. aeruginosa (DSM 1117) were used as references (German Collection of Microorganisms and Cell Cultures, DSMZ, Braunschweig, Germany). In addition, for each species, 12 
randomly collected clinical isolates were provided by the Institute for Medical Microbiology of the University Medical Centre Göttingen (see Table 1).

Each sample was grown on Columbia sheep agar plates (Ref. 43041; BioMérieux, Nürtingen, Germany) and then transferred into LB. For long-term storage, all strains were stored at $-80^{\circ} \mathrm{C}$ as glycerol stocks (LB $w / v 55 \%$ glycerol).

Overnight cultures were inoculated from the glycerol stocks. On the next morning, each culture was diluted down to $1: 1,000$ and transferred into a 2,000-ml Schott flask to start the actual study culture. Overnight cultures were diluted in order to ensure similar conditions for plotting of bacterial growth curves by measuring the optical density (OD) at a wavelength of $600 \mathrm{~nm}$. All cultures were left constantly moving at a temperature of $37{ }^{\circ} \mathrm{C}$. OD was measured for the plain LB medium, the overnight culture, at the beginning of the growing culture $(0 \mathrm{~min})$ and during the growth of the cultures. A growth curve was created for each bacterial culture on the basis of OD measurements. Finally, a sample from each culture was grown for $72 \mathrm{~h}$ on Columbia sheep agar plates and verified by MALDI-TOF MS fingerprinting using the Bruker BioTyper 3.0 system (Bruker Daltonics, Bremen, Germany).

\section{Headspace sampling}

MCC-IMS measurements were performed at four time points during the growth of the cultures, representing four phases of the bacterial growth curve [lag phase (lag), early logarithmic phase $(\log 1)$, late logarithmic phase $(\log 2)$, stationary phase (stat)]. E. coli cultures were measured 45, 105, 225 and $405 \mathrm{~min}$, and $P$. aeruginosa cultures were measured after 90, 270, 440 and $760 \mathrm{~min}$ after inoculation. Each measurement was performed 12 times for the two reference

Table 1 Bacterial reference strains and origins of the clinical isolates used for this study

\begin{tabular}{lll}
\hline & Escherichia coli & Pseudomonas aeruginosa \\
\hline Reference strain & DSM 25944 & DSM 1117 \\
Clinical isolate 1 & Blood culture & Tracheal aspirate \\
Clinical isolate 2 & Bronchial aspirate & Wound swab lower leg \\
Clinical isolate 3 & Wound swab & Swab ear \\
Clinical isolate 4 & Urine culture & Swab lower leg \\
Clinical isolate 5 & Urine culture & Wound swab upper leg \\
Clinical isolate 6 & Urine culture & Urine culture \\
Clinical isolate 7 & Swab abdomen & Wound swab \\
Clinical isolate 8 & Swab abscess & Swab kidney,intraoperative \\
Clinical isolate 9 & Tracheal aspirate & Deep swab abscess \\
Clinical isolate 10 & Blood culture & Urine culture \\
Clinical isolate 11 & Deep swab groin & Wound swab shoulder \\
Clinical isolate 12 & Urine culture & Blood culture \\
\hline
\end{tabular}

strains. For each of the 12 clinical isolates, one measurement was performed at each growth phase.

For the headspace analyses, each culture flask was attached to the MCC-IMS using a two-way (in- and outlet) screw cap. In order to minimize environmental contamination of the sample during measurement, a constant flow of $100 \mathrm{ml} / \mathrm{min}$ of synthetic air was supplied via the gas inlet of the screw cap, and the gas outlet was attached to MCC-IMS. Gas samples were taken by activating the MCC-IMS's vacuum pump (pump flow $500 \mathrm{ml} / \mathrm{min}$ ) for $10 \mathrm{~s}$. After sampling was completed, the culture flask was disconnected and incubation continued for further measurements. In order to clean the sampling loop after each measurement, a number of humid air measurements were performed.

\section{Multi-capillary column-ion mobility spectrometer}

The type and specification of the MCC-IMS device used for this study are summarised in Table 2. The principle of gas sample analyses by the MCC-IMS technology has been described in detail previously (Baumbach 2009). We therefore only give a short overview on its principle: After the gas sample has passed the sample loop, a sixport valve (Valco Instruments Inc. Co. and VICI AG, Schenkon, Switzerland) enables its introduction into the multi-capillary column. After preseparation, the gas samples are led into the ionization chamber, where they are ionized through charge transfer from prior ionized reactant ions. Drift region and ionisation region are separated by a shutter grid that is opened for defined range of time.

Table 2 Specifications of the MCC-IMS used for the study

MCC-IMS device

Preseparation

MCC temperature

Sample loop

Ionization source

Electric field strength

Shutter opening time

Drift and carrier gas

Drift gas flow

Carrier gas flow

Temperature

Pressure

Tubing

Fittings
BioScout, B\&S Analytik $\mathrm{GmbH}$, Dortmund, Germany

Multi-capillary column OV-5 (length $22 \mathrm{~cm}$ ), Multichrom Ltd., Novosibirsk, Russia

$40{ }^{\circ} \mathrm{C}$

Stainless steel, volume $10 \mathrm{ml}$ B-radiation, ${ }^{63} \mathrm{Ni}(550 \mathrm{MBq})$ $330 \mathrm{~V} / \mathrm{cm}$

$300 \mu \mathrm{s}$

Synthetic air, Air Liquide AG, Düsseldorf, Germany

$100 \mathrm{ml} / \mathrm{min}$

$150 \mathrm{ml} / \mathrm{min}$

Ambient

Ambient

PTFE, Bohlender GmbH, Grünsfeld, Germany

$1 / 8$ in. Swagelock Co., Solon, USA 
Ionized molecules pass the shutter grid and get accelerated towards the detector (Faraday plate) along the drift region of the MCC-IMS. On their way to the detector, ions are separated through collision with drift gas molecules moving in the opposite direction, with their individual velocities depending on size and shape of the molecules. Drift times are measured, and the respective drift velocities are calculated for the known drift distances. Ion mobility is calculated by normalizing the drift velocities to the known electrical field. In a last step, the reduced ion mobility, which is characteristic to an ion, is calculated by normalization to temperature and pressure (Vautz et al. 2009).

In the drift region, ions are moving through an external electric field, which can be charged positively or negatively. To ensure a maximum reliability, we decided to focus our resources on multiple and reproducible measurements using the positive mode of the MCC-IMS only. Thus, signals that might have been detectable by using the negative mode were neglected in the study.

Depending on concentration of substances, clusters of more than one ion can occur. Thus, the ionization of a substance by a $\mathrm{NI}^{63}$ source can result in a single signal in the IMS plot (monomer) or lead to the formation of more than one signal at the same retention time (dimer or even trimer; Borsdorf and Eiceman 2006). Monomer, dimer and trimer have the same retention times but show increasing inverse ion mobilities (see below).

\section{MCC-IMS data analyses}

Analyses of the detected MCC-IMS signals were performed using the VisualNow software (version 1.2, B\&S Analytik GmbH, Dortmund, Germany). The software visualizes MCC-IMS data as a three-dimensional matrix in which the $x$-axis indicates the inverse ion mobility in volt seconds per square centimetre and the $y$-axis indicates the MCC retention time in seconds. In the resulting topographic plot, the matrix values indicate the signal intensity in volts. In order to normalize all MCCIMS data, alignment was performed according to Vautz et al. (2009) and Perl et al. (2010). The MCC-IMS signals were identified by comparing the detected signals with a database, including the reference data for 125 substances. For each substance, reference measurements were performed prior to this study as it has been described before (Jünger et al. 2012). The IUPAC names of the substances were used in this study. Signals we were not able to identify were named according to their position in the 2D topographic plot as " $P x y$ ", whereas " $x$ " is representing the inverse ion mobility $x 1,000$ and " $y$ " is representing the $\mathrm{MCC}$ retention time (e.g. P 574 118= $\left.0.574 \mathrm{Vs} / \mathrm{cm}^{2} ; 118 \mathrm{~s}\right)$.
Changes in signal intensities of the detected MCC-IMS signals were followed during the growth curve of the bacteria using the four measurements as described above. Statistical plots and analyses were performed by using a statistical software (Statistica 10, StatSoft Inc., Tulsa, USA).

\section{Results}

Bacterial growth behaviour

For both bacteria analysed in this study, reproducible growth curves could be established by the methods described above. The typical bacterial growth behaviour (including lag phase, logarithmic growth phase and stationary phase) was observed for all reference strains and clinical isolates. There were no obvious variations between the growth curves of different strains of one species (see Fig. 1, the points in time of IMS measurements are indicated). While $P$. aeruginosa cultures reached the static growth phase approximately $700 \mathrm{~min}$ after inoculation from overnight cultures, $E$. coli cultures grew faster and showed static growth behaviour from approximately $300 \mathrm{~min}$ after inoculation. MALDI-TOF MS fingerprinting confirmed the identity of the bacteria in all cultures.

\section{Determination of bacterial volatile patterns}

Changes in signal intensity were observed in 19 areas of the IMS plot. Thirteen of these peaks (2-Propanone, Azane [dimer], Decan-1-ol, Dodecane, Ethanol, 2-Ethylhexan-1ol, Indole, Octan-1-ol [monomer and dimer], P_603_25, P_648_36, P_755_105, P_778_4) occurred or changed their signal intensities only over one of the investigated bacteria. Therefore, these compounds were considered to be suitable for identifying the respective bacteria in the setting of this study.

One particular peak (P_642_48) changed its signal intensity in both species, but at different times during their growth. In E. coli, P_642_48 reached its highest signal intensity during the early logarithmic phase (log1 phase) and showed a decreasing trend in the following growth phases. For $P$. aeruginosa, the signal intensity of P_642_48 increased constantly, but did not reach the level that was detected over the E. coli cultures.

The five remaining peaks (Azane [monomer], 5-Methylheptan-3-on, Nonanal, 2-Phenylacetaldehyde, $P$ 720_16) occurred or changed their signal intensities similarly over both $E$. coli and $P$. aeruginosa cultures, and no obvious differences were observed (see Fig. 2). In one of these five unspecific VOCs (Azane [monomer]), the signal intensity increased over the growing cultures. The remaining four signals all showed decreasing trends. 
Fig. 1 Growth curves of E. coli and $P$. aeruginosa cultures with data points indicating the mean OD and whiskers indicating the 5-95\% confidence interval of OD at each point of measurement. The points in time of MCC-IMS measurements along the growth curve are indicated by arrows
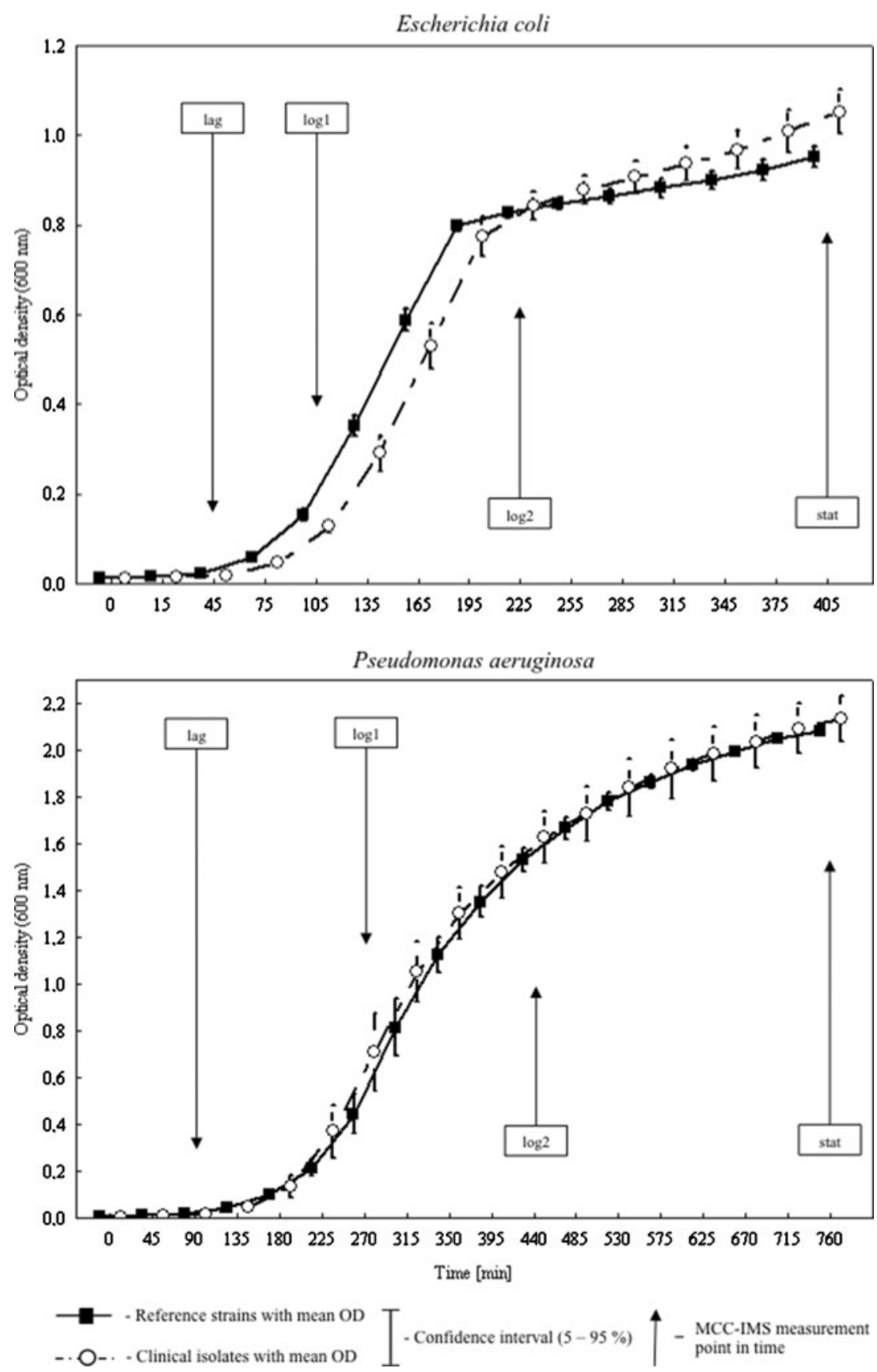

Figure 3 shows whether a peak was considered to be helpful for the differentiation of the two bacteria or not. It also shows the positions of the signals in a twodimensional topographic plot. The names of the substances and peak areas detected in this study are listed in Table 3.

Over the E. coli cultures, six signals (Decan-1-ol, Ethanol, Indole, Octan-1-ol [monomer and dimer], P 755 105) were detected (see Fig. 4) that were helpful for its differentiation in a complex fluid medium such as LB. All signals newly occurred during the growth of $E$. coli or distinctly increased from the baseline. The signal intensities of all six VOCs started to change during the logarithmic growth phase. We were not able to detect any obvious changes in the early growth phases (lag, log1) but observed reproducible increases starting with the late logarithmic phase $(\log 2)$. Except for Indole, which showed a constantly increasing trend, we detected a decrease in the signal intensities for these VOCs during the static growth phase, which could indicate the beginning of bacterial death due to overpopulation and expiring nutrients in the medium.

Over $P$. aeruginosa cultures, seven peaks (2-Propanone, Azane [dimer], Dodecane, 2-Ethylhexan-1-ol, P_603_25, P_648_36, P_778_4) were detected that enabled 

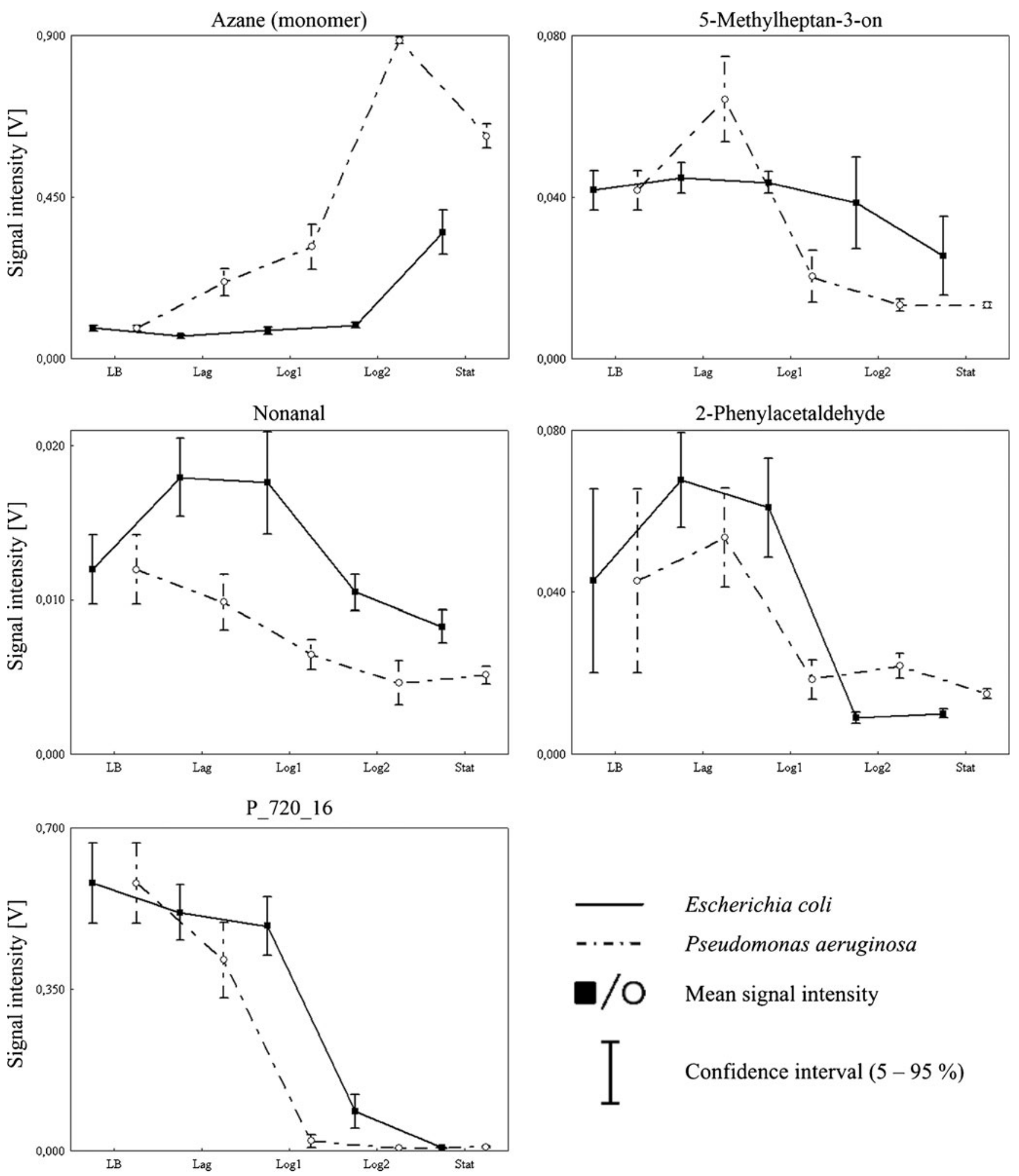

Fig. 2 Substance names and trends in signal intensity of all analysed peaks that were not specific for one of the two bacteria

differentiation between the two bacteria in the setting of this study (see Fig. 5). For six VOCs, we found increasing trends in signal intensity during the growth of $P$. aeruginosa. These included 2-Propanone, which showed a quick increase starting in the lag phase. But after reaching its maximum in the early logarithmic growth phase $(\log 1)$, our results showed a decreasing trend in the late growth phases that reached the baseline in the static phase (stat). For the 
Fig. 3 Two-dimensional topographic plot with the positions of 19 peaks that were analysed for this study, numbered in alphabetical order. Data labels give information on whether a particular area was considered specific for one of the bacteria or not. Peaks are listed in Table 2

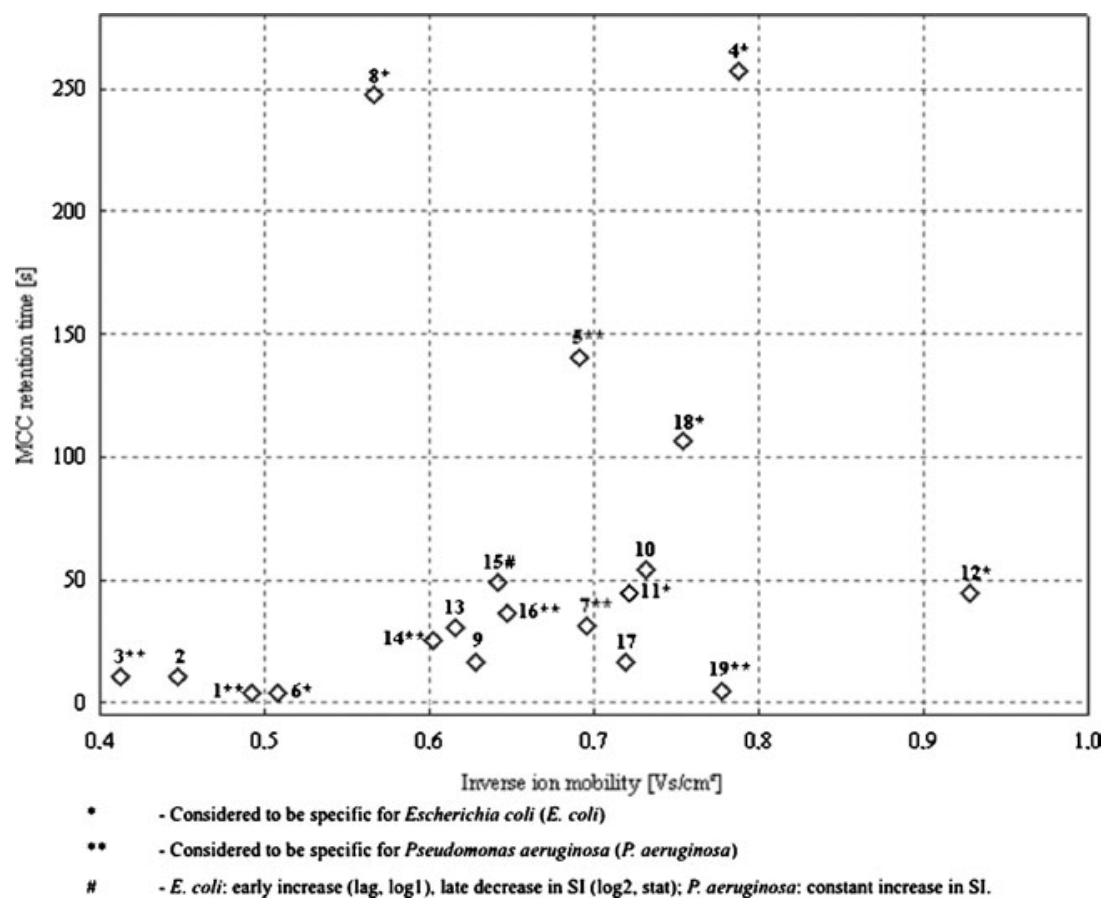

remaining five peaks (Azane [dimer], Dodecane, p_603 25, P_648_36, P_778_4), the signal intensities began to rise during the late logarithmic and static growth phase. While

Table 3 All peaks analysed for this study with their matching substance names and their coordinates in the topographical MCC-IMS plot, with the inverse ion mobility $[1 / \mathrm{k} 0$ (volt seconds per square centimetre)] and the MCC retention time [RT (seconds)], as seen before in Fig. 3

\begin{tabular}{llll}
\hline Peak no. & Substance name (IUPAC) & $1 / \mathrm{k} 0$ & RT \\
\hline 1 & 2-Propanone & 0.493 & 3.9 \\
2 & Azane (monomer) & 0.448 & 10.5 \\
3 & Azane (dimer) & 0.413 & 10.5 \\
4 & Decan-1-ol & 0.788 & 256.6 \\
5 & Dodecane & 0.692 & 140.0 \\
6 & Ethanol & 0.509 & 3.7 \\
7 & 2-Ethylhexan-1-ol & 0.696 & 31.0 \\
8 & Indole & 0.567 & 247.2 \\
9 & 5-Methylheptan-3-on & 0.629 & 16.0 \\
10 & Nonanal & 0.732 & 53.5 \\
11 & Octan-1-ol (monomer) & 0.722 & 44.3 \\
12 & Octan-1-ol (dimer) & 0.929 & 44.3 \\
13 & 2-Phenylacetaldehyde & 0.616 & 30.4 \\
14 & P_603_25 & 0.603 & 25.0 \\
15 & P_642_48 & 0.642 & 48.3 \\
16 & P_648_36 & 0.648 & 36.0 \\
17 & P_720_16 & 0.720 & 16.0 \\
18 & P_755_105 & 0.755 & 106.0 \\
$\mathrm{cb}$ & P_778_4 & 0.778 & 4.0 \\
\hline & & & \\
& & &
\end{tabular}

the signal intensity of $\mathrm{P} 603 \_25$ reached its maximum in the $\log 2$ phase and showed a decrease in the stat phase, the increasing trends of the other four VOCs continued through all measurements. The signal intensity of 2-Ethylhexan-1-ol over $P$. aeruginosa showed a decreasing trend during its growth, starting in the lag phase.

Except for two early changing signals in the headspace over growing $P$. aeruginosa cultures, we detected obviously different VOC patterns in the late growth phases (log2 and stat phase). Figures 2, 4 and 5 show trends in signal intensity of all peaks analysed for this study.

Volatile compounds were detected in the headspace over growing bacteria cultures of reference strains as well as randomly collected clinical isolates of different origin. There was no obvious difference in the analysed peaks between reference strain and clinical isolate cultures.

\section{Discussion}

In the present study, we describe changes of volatile organic compounds (VOCs) in the headspace over two human pathogenic bacteria during their growth in LB. We performed MCC-IMS analyses over cultures of $E$. coli and $P$. aeruginosa at four time points representing the characteristic stages of the bacterial growth curve.

Yet little is known on how the production and detection of microbial VOCs is influenced by the concentration of pathogens in a tissue or a growth medium. Thus so far, no information is available from which time point on bacteria can be identified by headspace analyses. Only a few studies 

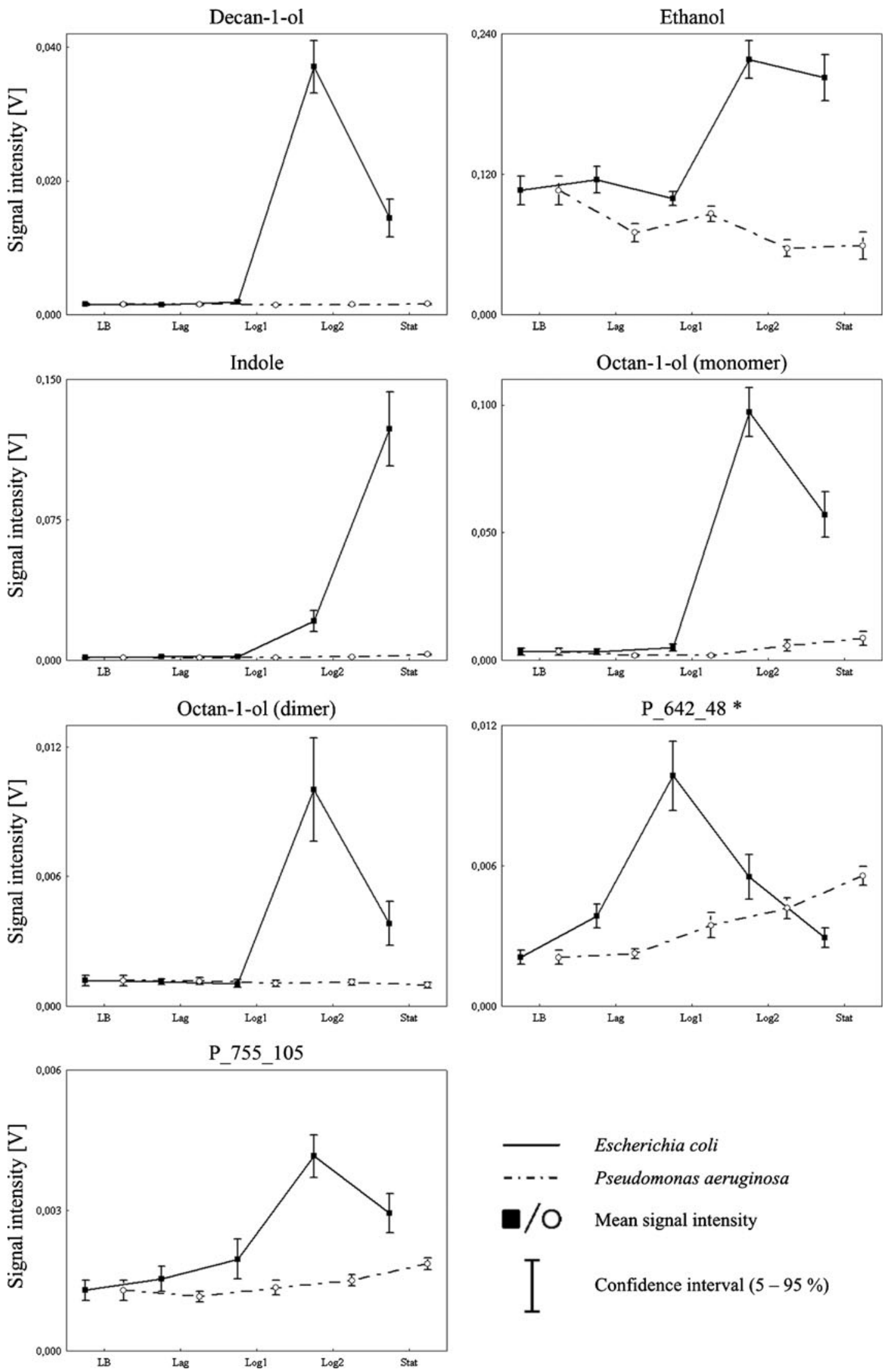

Escherichia coli

-.- Pseudomonas aeruginosa

$\square / O \quad$ Mean signal intensity

I Confidence interval (5-95\%)

Fig. 4 Substance names and trends in signal intensity of all peaks that were considered to be specific for E. coli. Peak P_642_48 (*) changed its signal intensity in both species, but at different times during their growth. However, it reached its maximum signal intensity over $E$. coli cultures 

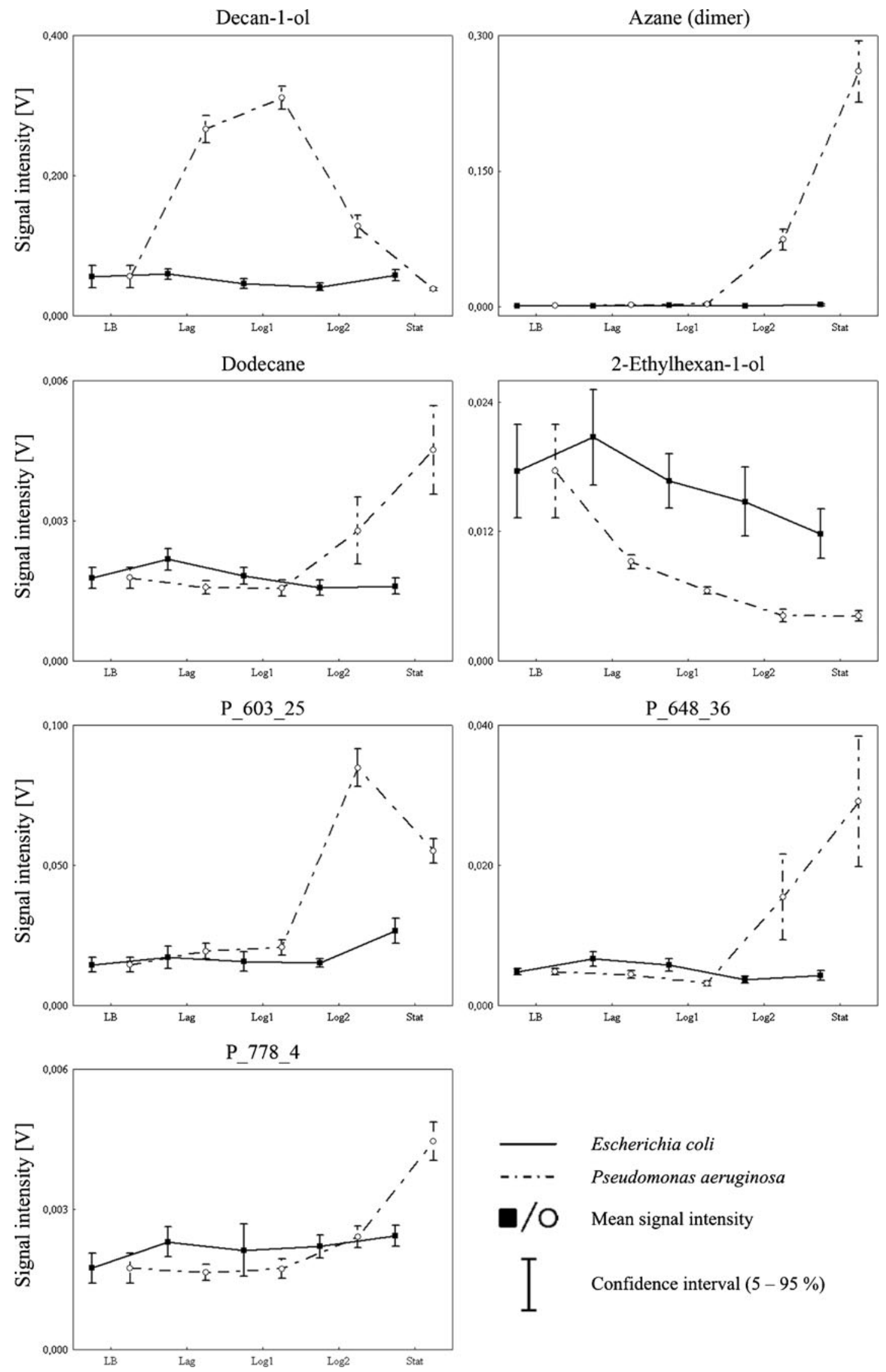

Fig. 5 Substance names and trends in signal intensity of all peaks that were considered to be specific for $P$. aeruginosa 
have addressed this issue (O'Hara and Mayhew 2009; Maddula et al. 2009). In 2009, Maddula et al. published a study in which they focused on the VOC emissions over cultures of $E$. coli as a function of time (Maddula et al. 2009). They detected four different newly occurring metabolites over the growing cultures and observed a "biomass dependent" increase in signal intensity for them. However, in order to eliminate most of the volatile background caused by a complex growth medium, they used a minimal medium optimized for the (little) metabolic needs of E. coli. Such individual settings, tailored for each microbe, obviously will not be suitable for diagnostic purposes in medical practice as it is the aim of such a setting to identify the latter. Therefore, a universal growth medium is required. Besides ethanol, we were unable to reproduce the signals observed by Maddula et al. in the complex growth medium. Therefore, we observed five different signals (Decan-1-ol, Indole, Octan-1-ol [monomer and dimer], P_755_105) that might be helpful for the identification of $E$. coli.

Our results partly match the recent findings of Jünger et al., in which they analysed the headspace over several bacterial cultures on Columbia sheep blood agar plates, including E. coli and P. aeruginosa (Jünger et al. 2012). Since they incubated agar plates $24 \mathrm{~h}$ before measuring the headspace, no further information was given on the timing of the occurring VOCs. But cultures have been measured in this setting during one of the late growth phases. However, both studies detected ethanol and indole over E. coli.

For $P$. aeruginosa, we observed six VOCs (2-Propanone, Azane [dimer], Dodecane, 2-Ethylhexan-1-ol, P_603_25, P 648_36, P_778_4), whereas Jünger et al. were able to detect two newly occurring substances (ethanol and phenethyl alcohol) in the positive ion mode. In our study, neither of these two VOCs showed an increasing trend in signal intensity over $P$. aeruginosa. Due to the small differences in the detection of the two MCC-IMS devices used for the two studies, we suggest that P_778_4 (present study) and p_774_4 (Jünger et al. 2012) match each other. This peak showed an increase in signal intensity particularly over $P$. aeruginosa in the late logarithmic and static growth phases. Compared to plain LB, we observed slightly higher signal intensities over E. coli for P_778_4 as well (see Fig. 5).

As demonstrated by O'Hara and Mayhew (2009), the growth medium has a certain influence on the occurrence of VOCs over bacterial cultures. They compared the emissions over cultures of a Staphylococcus aureus strain during its growth in three different media. Although they were unable to observe any changes in the emitted VOCs, there were changes in the timing of their occurrence, as well as changes in the signal intensities of the detected substances. Our choice for LB as a medium might therefore explain the differences in the results of the detected VOC patterns
(Jünger et al. 2012; Maddula et al. 2009; O'Hara and Mayhew 2009).

The composition of a standardised medium changes during the growth phases due to consumption of substrates during exponential growth and resulting lack of nutrients in the static growth phases. As a consequence of this dynamic process, changes in the detected VOCs and/or their signal intensities might occur. That could explain the late decrease of the signal intensities in some of the VOCs in our study. Nevertheless, for the two investigated bacteria, at least in the late logarithmic phase, several indicator metabolites for the species can be described.

LB was chosen as growth medium as it is easy to handle and allows culturing of many human pathogenic microbes, including bacteria and fungi. Although a number of VOCs are emitted from LB itself, we were able to detect different VOC patterns for both bacteria investigated. Our study setting permitted a differentiation between $E$. coli and $P$. aeruginosa at least at the beginning of the late logarithmic growth phase ( $\log 2$ phase). The continuing characterization of pathogens using MCC-IMS analyses is a necessary step on the method's way to a clinical use. Regarding the current state of knowledge, using one medium only might prevent us from comparing apples with oranges.

We preferred the use of a fluid medium because the growth phases can be understood more easily than by culturing on a solid medium. That might be of particular interest for considering the use of MCC-IMS analyses in direct clinical sampling. Our results show that most of the signals assumed to be helpful for the identification of $E$. coli (e.g. Octan-1-ol, Indole) and P. aeruginosa (e.g. Azane [dimer], P_603_25) occur or change their signal intensities in the late logarithmic and static growth phases. Measuring the headspace over a fully grown (after $24 \mathrm{~h}$ or later) agar plate culture neglects that information (Jünger et al. 2012; Perl et al. 2011). This could lead to the misinterpretation that a particular metabolite must be detectable over every microbial culture grown on the same medium, regardless of which growth phase the culture is in at the moment of measurement. This fact implies serious challenges for the possibility of direct clinical sampling, e.g. direct breath sampling in pulmonary infections, as little is known about bacterial growth behaviour in vital lung tissue and its influence on the emission of VOCs.

We were able to identify several of the detected signals. Due to the use of different media, our results partly differ from those of other studies. However, according to the review of Schulz and Dickschat (2007) on bacterial VOC emissions, most of the substances we identified are known indicators for bacterial growth. These substances contain aromatic compounds (Indole, 2-Phenylacetaldehyde), fatty acid derivates (Decan-1-ol, Nonanal, Octan-1-ol), ketones (2-Propanone [Acetone]) and Azane (Ammonia), as a 
simple nitrogen-containing compound emitted by many different bacteria. Furthermore, we observed the formation of dimers using the positive ion mode of the MCC-IMS. As described by Borsdorf and Eiceman (2006), the ionization of a substance by a $\mathrm{Ni}^{63}$ source can lead to the formation of monomers and dimers (or even trimers) in an IMS plot, with dimers implying a high amount of that particular compound in the gas sample. In the present study, we observed dimer formation for Azane (Ammonia), Indole and Octan-1-ol. Indole and Octan-1-ol occurred over E. coli only, whereas Azane (Ammonia) occurred over both species. Interestingly, we were only able to detect the dimer of Azane during the late growth phases of $P$. aeruginosa. We therefore considered the dimer of the substance as potentially being helpful for identifying $P$. aeruginosa in our particular setting.

In our study, we compared the VOC patterns over human pathogenic reference strains of $E$. coli and $P$. aeruginosa with the VOC patterns that occurred in the headspaces over cultures of 12 randomly collected clinical isolates of the same bacteria. We were unable to detect any differences in the signal intensity trends of the peaks analysed. Our findings support the suggestion that the VOCs detected over one bacterial strain can be transferred to other strains of the same species. Nevertheless, more research is needed to provide evidence for other microbes. For medical research, it seems to be preferable to focus on human pathogenic strains for characterization because some VOCs might represent pathogenic characteristics of the investigated microbes. For example, Indole was found to regulate biofilm formation in $E$. coli (Martino et al. 2003), but was not present over the nonpathogenic recombinant $E$. coli BL21 (Maddula et al. 2009). Hereby the influence of the medium chosen for growing $E$. coli remains unclear, and this assumption should be investigated in future studies.

The use of human pathogenic bacteria strains grown in a universal fluid medium is a strength of our study as this setting provides the opportunity to build up a comparable stock of data on a maximum number of microbes. To ensure the reliability of our analyses, we measured 12 cultures of each reference strain and validated the results by analysing 12 clinical isolates of the identical species from different origins. This validation on randomly collected clinical isolates might additionally strengthen our study as it proves that the results of one bacterial strain are transferable to other strains of the same species.

For this study, we only used the positive ion mode of the MCC-IMS, which is a certain limitation. We might have detected more specific VOCs for the two bacteria by using the negative ion mode of the MCC-IMS as well. However, we focused our resources on ensuring maximum reliability of our results by providing multiple measurements. The analyses in the negative ion mode should be done in the future and, when performed in a similar setting, the results might complete the information provided by the present study.

The fact that no information is provided regarding the strains to which the clinical isolates were belonging makes the results of that part of the study less comparable and represents another limitation. Our results demonstrate that MCC-IMS analyses allow differentiation between $E$. coli and $P$. aeruginosa in a growing culture. The specific VOC patterns that enabled differentiation between the two species occurred during the late growth phases ( $\log 2$ and stat phase). Further investigations in a comparable setting are needed to ensure that identification of an unknown pathogen in clinical material is possible using this method. In addition, we were able to show that the VOC patterns of different human pathogenic strains of one bacterial species are identical.

In summary, these findings underline the potential of the method for fast and reliable microbial identification and gives further evidence for its diagnostic use as either a laboratory tool or even as an online bedside diagnostic tool (e.g. through direct breath sampling in ventilated patients). More research is needed to collect comparable information on the characteristic VOC patterns of a greater number of human pathogenic microbes and the timing of their occurrence. The present study represents one part of this puzzle and needs to be extended by a clinical study that investigates the reliability of the method in analyses of native clinical material.

Funding This work was part of the cooperation project KF 2111207AK0 and was founded by the Central Innovation Programme SME (ZIM). The financial support of the German Federal Ministry of Economics and Technology is gratefully acknowledged

Open Access This article is distributed under the terms of the Creative Commons Attribution License which permits any use, distribution, and reproduction in any medium, provided the original author(s) and the source are credited.

\section{References}

Baumbach JI (2009) Ion mobility spectrometry coupled with multicapillary columns for metabolic profiling of human breath. J Breath Res 3(3):034001. doi:10.1088/1752-7155/3/3/034001

Borsdorf H, Eiceman GA (2006) Ion mobility spectrometry: principles and applications. Appl Spectrosc Rev 41(4):323-375. doi:10.1080/05704920600663469

Chaim W, Karpas Z, Lorber A (2003) New technology for diagnosis of bacterial vaginosis. Eur J Obstet Gynecol Reprod Biol 111(1):83-87

Fenollar F, Goncalves A, Esterni B, Azza S, Habib G, Borg JP, Raoult D (2006) A serum protein signature with high diagnostic value in bacterial endocarditis: results from a study based on surfaceenhanced laser desorption/ionization time-of-flight mass spectrometry. J Infect Dis 194(10):1356-1366. doi:10.1086/508429

Garnacho-Montero J, Aldabo-Pallas T, Garnacho-Montero C, Cayuela A, Jimenez R, Barroso S, Ortiz-Leyba C (2006) Timing of adequate antibiotic therapy is a greater determinant of outcome than are TNF and IL-10 polymorphisms in patients with sepsis. Crit Care 10(4):R111. doi:10.1186/cc4995 
Houck PM, Bratzler DW, Nsa W, Ma A, Bartlett JG (2004) Timing of antibiotic administration and outcomes for Medicare patients hospitalized with community-acquired pneumonia. Arch Intern Med 164(6):637-644. doi:10.1001/archinte.164.6.637

Jünger M, Vautz W, Kuhns M, Hofmann L, Ulbricht S, Baumbach JI, Quintel M, Perl T (2012) Ion mobility spectrometry for microbial volatile organic compounds: a new identification tool for human pathogenic bacteria. Appl Microbiol Biotechnol 93(6):26032614. doi:10.1007/s00253-012-3924-4

Kai M, Haustein M, Molina F, Petri A, Scholz B, Piechulla B (2009) Bacterial volatiles and their action potential. Appl Microbiol Biotechnol 81(6):1001-1012. doi:10.1007/s00253-008-1760-3

Kollef MH, Sherman G, Ward S, Fraser VJ (1999) Inadequate antimicrobial treatment of infections: a risk factor for hospital mortality among critically ill patients. Chest 115(2):462-474

Kumar A, Ellis P, Arabi Y, Roberts D, Light B, Parrillo JE, Dodek P, Wood G, Kumar A, Simon D, Peters C, Ahsan M, Chateau D (2009) Initiation of inappropriate antimicrobial therapy results in a fivefold reduction of survival in human septic shock. Chest 136(5):1237-1248. doi:10.1378/chest.09-0087

Mach KE, Du CB, Phull H, Haake DA, Shih MC, Baron EJ, Liao JC (2009) Multiplex pathogen identification for polymicrobial urinary tract infections using biosensor technology: a prospective clinical study. J Urol 182(6):2735-2741. doi:10.1016/j.juro.2009.08.028

Maddula S, Blank LM, Schmid A, Baumbach JI (2009) Detection of volatile metabolites of Escherichia coli by multi capillary column coupled ion mobility spectrometry. Anal Bioanal Chem 394(3):791-800. doi:10.1007/s00216-009-2758-0

Martino PD, Fursy R, Bret L, Sundararaju B, Phillips RS (2003) Indole can act as an extracellular signal to regulate biofilm formation of Escherichia coli and other indole-producing bacteria. Can J Microbiol 49(7):443-449. doi:10.1139/w03-056

O'Hara M, Mayhew CA (2009) A preliminary comparison of volatile organic compounds in the headspace of cultures of Staphylococcus aureus grown in nutrient, dextrose and brain heart bovine broths measured using a proton transfer reaction mass spectrometer. J Breath Res 3(2):027001. doi:10.1088/1752-7155/3/2/027001

Perl T, Bodeker B, Jünger M, Nolte J, Vautz W (2010) Alignment of retention time obtained from multicapillary column gas chromatography used for VOC analysis with ion mobility spectrometry. Anal Bioanal Chem 397(6):2385-2394. doi:10.1007/s00216-010-3798-1

Perl T, Jünger M, Vautz W, Nolte J, Kuhns M, Borg-von Zepelin M, Quintel M (2011) Detection of characteristic metabolites of
Aspergillus fumigatus and Candida species using ion mobility spectrometry-metabolic profiling by volatile organic compounds. Mycoses 54(6):e828-e837. doi:10.1111/j.1439-0507.2011.02037.x

Ruzsanyi V, Baumbach JI, Sielemann S, Litterst P, Westhoff M, Freitag L (2005) Detection of human metabolites using multi-capillary columns coupled to ion mobility spectrometers. J Chromatography A 1084(1-2):145-151

Schulz S, Dickschat JS (2007) Bacterial volatiles: the smell of small organisms. Natural Product Reports 24(4):814-842. doi:10.1039/ b507392h

Shnayderman M, Mansfield B, Yip P, Clark HA, Krebs MD, Cohen SJ, Zeskind JE, Ryan ET, Dorkin HL, Callahan MV, Stair TO, Gelfand JA, Gill CJ, Hitt B, Davis CE (2005) Species-specific bacteria identification using differential mobility spectrometry and bioinformatics pattern recognition. Anal Chem 77(18):5930-5937. doi:10.1021/ac050348i

Smith GB, Eiceman GA, Walsh MK, Critz SA, Andazola E, Ortega E, Cadena F (1997) Detection of Salmonella typhimurium by handheld ion mobility spectrometer: a quantitative assessment of response characteristics. Field Anal Chem Tech 1(4):213-226

Snyder AP, Shoff DB, Eiceman GA, Blyth DA, Parsons JA (1991) Detection of bacteria by ion mobility spectrometry. Anal Chem 63(5):526-529

Stotzky G, Schenck S (1976) Volatile organic compounds and microorganisms. CRC Crit Rev Microbiol 4(4):333-382

van Veen SQ, Claas EC, Kuijper EJ (2010) High-throughput identification of bacteria and yeast by matrix-assisted laser desorption ionization-time of flight mass spectrometry in conventional medical microbiology laboratories. J Clin Microbiol 48(3):900-907. doi:10.1128/JCM.02071-09

Vautz W, Bödeker B, Baumbach JI, Bader S, Westhoff M, Perl T (2009) An implementable approach to obtain reproducible reduced ion mobility. Inter $\mathbf{J}$ for Ion Mobility Spectrometry 12(2):47-57. doi:10.1007/s12127-009-0018-9

Yoo SM, Lee SY (2008) Diagnosis of pathogens using DNA microarray. Recent Patents on Biotechnology 2(2):124-129

Zhu LX, Zhang ZW, Wang C, Yang HW, Jiang D, Zhang Q, Mitchelson K, Cheng J (2007) Use of a DNA microarray for simultaneous detection of antibiotic resistance genes among staphylococcal clinical isolates. J Clin Microbiol 45(11):3514-3521. doi:10.1128/ JCM.02340-06

Zoller HF, Clark WM (1921) The production of volatile fatty acids by bacteria of the dysentery group. J Gen Physiol 3(3):325-330 